Supporting Information for

\title{
Supramolecular Hexagonal Platelet Assemblies with Uniform and Precisely-Controlled Dimensions
}

Gangfeng Chen, ${ }^{1}$ Guangpu Zhang, ${ }^{1}$ Bixin Jin, ${ }^{1}$ Mingyan Luo, ${ }^{1}$ Yunjun Luo, ${ }^{* 1,2}$ Satoshi Aya ${ }^{3}$ and Xiaoyu $\mathrm{Li}^{*} 1,2$

1 School of Materials Science and Engineering, Beijing Institute of Technology, Beijing 100081, China 2 Key Laboratory of High Energy Density Materials, Ministry of Education, Beijing Institute of Technology, Beijing 100081, China

3 RIKEN Centre for Emergent Matter Science, Saitama 351-0198, Japan

*Correspondence to: yjluo@bit.edu.cn; xiaoyuli@bit.edu.cn. 


\section{Table of Contents}

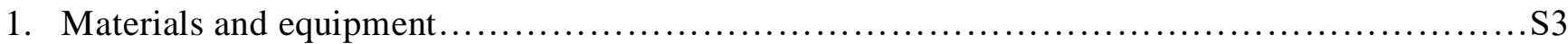

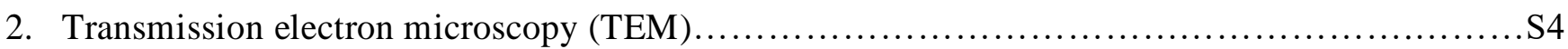

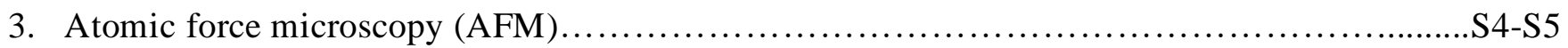

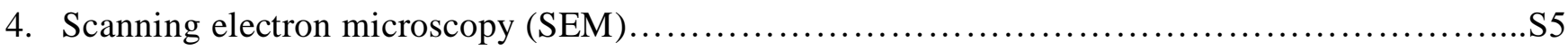

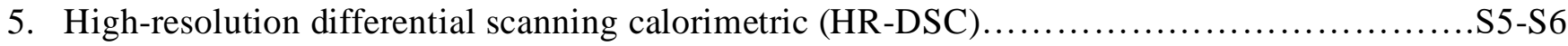

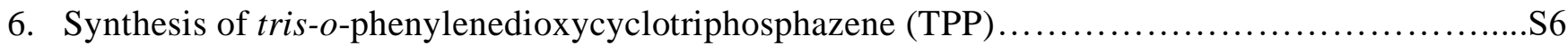

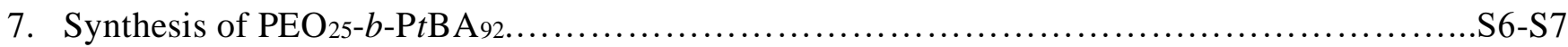

8. Preparation of non-uniform hexagonal platelets of P-E-P/TPP, BA-E/TPP and D-B/TPP .........S7

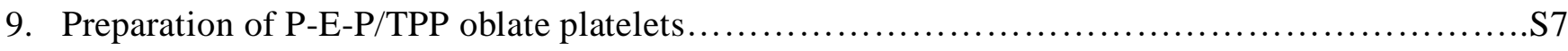

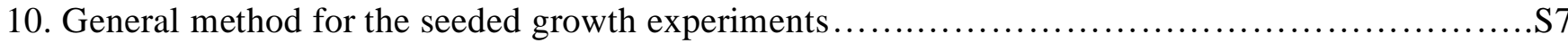

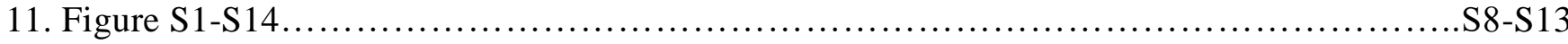

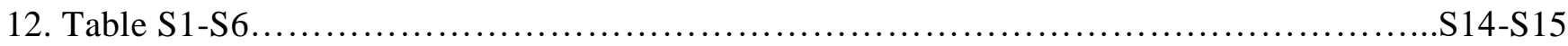

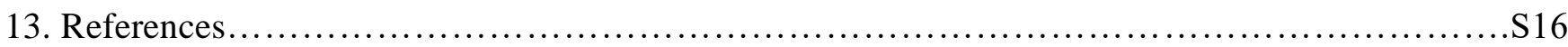




\section{Materials and equipment}

Poly(dimethyl siloxane)- $b$-polybutadiene diblock copolymer was purchased from Polymer Source Inc. Poly(propylene oxide)- $b$-poly(ethylene oxide)- $b$-poly(propylene oxide) triblock copolymer and other chemicals were purchased from Aldrich, as used as received.

${ }^{1} \mathrm{H}$ NMR spectra were recorded using Avance 500 (Bruker A.G.) instrument (operating at $500 \mathrm{MHz}$ ) at $25^{\circ} \mathrm{C}$.

Molecular weights and polydispersity indexes $\left(M_{w} / M_{n}\right)$ of polymers were obtained by gel permeation chromatography (GPC) using a Viscotex GPC max Chromatograph equipped with styrene/divinylbenzene columns with pore sizes of $500 \AA$ and $100000 \AA$, VE 3580 refractometer, and the UV-vis signal was monitored at $440 \mathrm{~nm}$. Butylated hydroxytoluene $(0.025 \%$, w.t.) stabilized THF was used as the eluent, with a flow rate of $1.0 \mathrm{~mL} / \mathrm{min}$. Samples were dissolved in the eluent $(2 \mathrm{mg} / \mathrm{mL})$ and filtered (Acrodisc, PTFE membrane, $0.45 \mathrm{~mm}$ ) before analysis. The calibration of refractive index detector was carried out using polystyrene standards (Viscotek).

Differential scanning calorimetry (DSC) were performed on a TA Instruments Q100 calorimeter at a scan rate of $10{ }^{\circ} \mathrm{C} / \mathrm{min}$ under nitrogen $(50 \mathrm{~mL} / \mathrm{min})$.

Powder wide-angle X-ray scattering (WAXS) analysis was performed at $25{ }^{\circ} \mathrm{C}$ using a Brucker D8 Advanced X-ray diffraction measurement system operated at $40 \mathrm{kV}$ and $40 \mathrm{~mA}$ with $\mathrm{Cu} \mathrm{K} \alpha$ radiation $(\lambda$ $=0.154 \mathrm{~nm})$. The data were collected at a scan rate of $0.1^{\circ} \mathrm{s}^{-1}$. The powder of TPP crystals and hexagonal platelets were collected by drying from their toluene solution.

Dynamic light scattering $\left(173^{\circ}\right)$ experiments were performed using a nano series Malvern zetasizer instrument equipped with a $633 \mathrm{~nm}$ red laser. Samples were analyzed in $1 \mathrm{~cm}$ glass cuvettes at $25^{\circ} \mathrm{C}$. For the purpose of the light scattering studies the refractive index of the platelets involved was assumed to be 1.60. The results of dynamic light scattering studies are reported as apparent hydrodynamic radius ( $R_{h}$,app), acknowledging that the particles have been modelled as hard spheres in the experiments conducted.

Regular bright field optical images were taken using an Olympus OLS5000 3D measuring laser microscope at $25^{\circ} \mathrm{C}$. 


\section{Transmission electron microscopy (TEM)}

The samples for electron microscopy were prepared by drop-casting one drop $(\sim 4.5 \mu \mathrm{L})$ of the solution onto a carbon coated copper grid (Beijing Zhongjingkeyi Technology Co., Ltd, mesh 230). Grids were placed on a piece of filter paper in advance to quickly remove excess solvent in $1 \mathrm{~s}$ to prevent further morphological change. Bright-field transmission electron microscopy (TEM) micrographs were obtained on a Hitachi $\mathrm{H}-7650 \mathrm{~B}$ microscope operating at $80 \mathrm{kV}$. No staining was applied for TEM samples. Images were analyzed using the Image-Pro Plus 6.0 software, which is free and available online. As the platelets reported here are trapped kinetically because of the crystallization of TPP, the morphologies observed from dried samples by TEM are anticipated to match closely those observed in solution. For the statistical length and angle analysis, more than 200 platelets in several images were analyzed by the software in order to obtain the side length and the interior angle information. The number average oblate platelet diameter or hexagonal platelet side length $\left(L_{n}\right)$ and weight average oblate platelet diameter or hexagonal platelet side length $\left(L_{w}\right)$ were calculated using Eqs. 1 and $2\left(L_{i}\right.$, the contour lengths; $N_{i}$, the number of length $L_{i} ; \mathrm{n}$, the number of lengths examined in each sample). The number average platelet interior angles $\left(A_{n}\right)$ and weight average platelet interior angles $\left(A_{w}\right)$ were calculated using Eqs. 3 and $4\left(A_{i}\right.$, the contour interior angles; $N_{i}$, the number of interior angles $A_{i} ; \mathrm{n}$, the number of interior angles examined in each sample). The standard deviations $(\sigma)$ of the measured side lengths or interior angles were calculated
(1) $L_{n}=\frac{\sum_{i=1}^{n} N_{i} L_{i}}{\sum_{i=1}^{n} N_{i}}$
(2) $L_{w}=\frac{\sum_{i=1}^{n} N_{i} L_{i}^{2}}{\sum_{i=1}^{n} N_{i} L_{i}}$
(3) $A_{n}=\frac{\sum_{i=1}^{n} N_{i} A_{i}}{\sum_{i=1}^{n} N_{i}}$
(4) $A_{w}=\frac{\sum_{i=1}^{n} N_{i} A_{i}^{2}}{\sum_{i=1}^{n} N_{i} A_{i}}$

directly from the data set.

\section{Atomic force microscopy (AFM)}

AFM experiments were conducted directly on the carbon-coated copper grid used for TEM analysis. AFM images were recorded with a Cypher ES (Asylum Research, CA, USA). The images of the hexagonal platelet were acquired in tapping mode in ambient environment. For the height analysis, more than 200 platelets in a number of images were analyzed by the software in order to obtain the height information. The number average platelet height $\left(H_{n}\right)$ and weight average platelet height $\left(H_{w}\right)$ were 
calculated using Eqs. 5 and $6\left(H_{i}\right.$, the contour height; $N$, the number of height $H_{i}$; n, the number of heights examined in each sample). The standard deviations $(\sigma)$ of the measured heights were calculated directly from the data set.

$$
\text { (5) } H_{n}=\frac{\sum_{i=1}^{n} N_{i} H_{i}}{\sum_{i=1}^{n} N_{i}} \quad \text { (6) } H_{w}=\frac{\sum_{i=1}^{n} N_{i} H_{i}^{2}}{\sum_{i=1}^{n} N_{i} H_{i}}
$$

The number average platelet volume $\left(V_{n}\right)$ and weight average platelet volume $\left(V_{w}\right)$ were calculated using Eqs. 7 and 8. The standard deviations $(\sigma)$ of the volumes were calculated from those of the measured side lengths and heights.
(7) $V_{n}=\frac{3}{2} \sqrt{3} L_{n}^{2} H_{n}$
(8) $V_{w}=\frac{3}{2} \sqrt{3} L_{w}^{2} H_{w}$

\section{Scanning electron microscopy (SEM)}

SEM experiments were conducted directly on the carbon-coated copper grid used for TEM analysis. SEM images were recorded by using a Hitachi SU8020 microscope operating at 30kV. An ultrathin coating of $\mathrm{Au}(\sim 5 \mathrm{~nm})$ was deposited via high vacuum evaporation.

\section{High-resolution differential scanning calorimetric (HR-DSC)}

To study the crystalline phase transition of the inclusion complex systems in solution state, we carried out a heat-capacity investigation by using a home-made HR-DSC instrument. The sample solutions were loaded into an aluminum cup and hermetically sealed with an aluminum lid. The mass of samples was fixed to be $18 \mathrm{mg}$ for the measurements. In the measurements, a heat flux type HR-DSC, equipped with semiconducting thermoelectric modules, was used to measure the temperature difference in a sample cell and a reference cell as a function of temperature of DSC chamber as in conventional DSC measurements, providing excellent temperature resolution of $<1 \mathrm{mK}$ and baseline stability. Thus, this technique allows us to measure thermal events with a high sensitivity of nano-watt level. Details of principle of the instrument and some applications are described elsewhere. ${ }^{1}$ All the measurements were performed with a super-slow scan rate of $\pm 0.01{ }^{\circ} \mathrm{C} / \mathrm{min}$ in order to put the samples as close as possible to their equilibrium state. 


\section{Synthesis of tris-o-phenylenedioxycyclotriphosphazene (TPP)}

TPP was prepared according to the literatures. ${ }^{2}$ A stirred mixture of hexachlorophosphazene $(4 \mathrm{~g}, 0.012$ $\mathrm{mol})$, catechol $(3.8 \mathrm{~g}, 0.035 \mathrm{~mol})$, and anhydrous sodium carbonate $(7.32 \mathrm{~g}, 0.069 \mathrm{~mol})$ was allowed to react in boiling tetrahydrofuran $(60 \mathrm{~mL})$ for $6 \mathrm{~h}$ under $\mathrm{N}_{2}$ atmosphere. The reaction mixture was then cooled to room temperature. The solid components were filtered off and then washed with aqueous $5 \%$ hydrochloric acid followed by distilled water. The insoluble residue was dried under vacuum for $6 \mathrm{~h}$ at $25^{\circ} \mathrm{C}$. Recrystallization in benzene yielded the TPP/benzene inclusion complex (IC). The guest-free TPP crystal was obtained by thermal treatment of the TPP/benzene IC at $75^{\circ} \mathrm{C}$ under vacuum for $8 \mathrm{~h}$. Yield: $2.8 \mathrm{~g}(54 \%)$. Anal. Calcd for $\mathrm{C}_{18} \mathrm{H}_{12} \mathrm{~N}_{3} \mathrm{P}_{3} \mathrm{O}_{6}: \mathrm{C} 47.08, \mathrm{H}$ 2.63, N 9.15. Found: C 50.76, H 3.16, N 8.41. MS: $460(\mathrm{~m} / \mathrm{z})$.

\section{Synthesis of $\mathrm{PEO}_{25}-b$-PtBA92}

PEO-OH (5.00 g, $2.50 \mathrm{mmol})$ and triethylamine $(0.250 \mathrm{~g}, 2.50 \mathrm{mmol})$ were dissolved in THF (20 mL), and 2-bromo-2-methylpropionyl bromide $(1.20 \mathrm{~g}, 5.00 \mathrm{mmol})$ was first dissolved in THF (10 $\mathrm{mL})$ and then added dropwise slowly. The reaction mixture was cooled in an ice/water bath and stirred at $0{ }^{\circ} \mathrm{C}$ for $2 \mathrm{~h}$ and then at ambient temperature for $24 \mathrm{~h}$. The filtered solution was concentrated, dissolved in dichloromethane, washed with aqueous $\mathrm{HCl}(0.3 \mathrm{~mol} / \mathrm{L})$ and water thrice, and then dried by anhydrous magnesium sulfate for $30 \mathrm{~min}$. The solvents were evaporated, and the raw product was precipitated in a large volume of diethyl ether twice. Final product $\mathrm{PEO}-\mathrm{Br}$ was obtained by recrystallization from methanol and dried in vacuum. Yield: $3.0 \mathrm{~g}(60 \%)$.

In a typical polymerization experiment, to a Schlenk tube PEO-Br (100 mg, $0.10 \mathrm{mmol}), \mathrm{CuBr}(14.34$ $\mathrm{mg}, 0.10 \mathrm{mmol})$, PMDETA (34.66 mg, $0.20 \mathrm{mmol})$, toluene $(1 \mathrm{~mL})$, and $t$ BA $(1.408 \mathrm{~g}, 11 \mathrm{mmol})$ were added under inert gas. The reaction mixture was heated at $60{ }^{\circ} \mathrm{C}$ for a desired period of time. Then the reaction was quenched in liquid nitrogen. The copper salt was removed by column chromatography $\left(\mathrm{Al}_{2} \mathrm{O}_{3}\right)$ using THF as the solvent and concentrated to ca. $10 \mathrm{~mL}$. polymer was recovered by precipitation into a mixture of methanol and water (1:1) with vigorous stirring and dried under vacuum. The purified 
$\mathrm{P} t \mathrm{BA}_{92}-b-\mathrm{PEO}_{25}$ was obtained as white solids. This polymer was characterization by GPC and ${ }^{1} \mathrm{H}$ NMR, as shown in Figure S6 and S7. GPC: $M_{\mathrm{n}}=12000, M_{\mathrm{w}} / M_{\mathrm{n}}=1.20$.

\section{Preparation of non-uniform hexagonal platelets of P-E-P/TPP, BA-E/TPP and D-B/TPP.}

To obtain the formation of the copolymer/TPP adducts, TPP and the copolymer were mixed at a suitable EO/TPP molar ratio of 1.3:1 or a suitable B/TPP molar ratio of 1:1 and ground in an agate mortar at room temperature for $2 \mathrm{~h}$ and then dispersed in toluene to form a $1 \mathrm{mg} / \mathrm{mL}$ solution. Then the solution was heated at $80^{\circ} \mathrm{C}$ with stirring for $1 \mathrm{~h}$ before the solution was allowed to cool down naturally to $20{ }^{\circ} \mathrm{C}$.

\section{Preparation of P-E-P/TPP oblate platelets.}

The solution of pristine P-E-P/TPP hexagonal platelets was diluted to $0.1 \mathrm{mg} / \mathrm{mL}$. The platelets were then sonicated (50W sonication processor equipped with a titanium sonotrode) at $25^{\circ} \mathrm{C}$ for $2 \mathrm{~h}$ (six 20 min periods).

\section{General method for the seeded growth experiments.}

For seeded growth, fresh solid mixture of polymer/TPP unimers $(\mathrm{EO} / \mathrm{TPP}=1.3$ or $\mathrm{B} / \mathrm{TPP}=1)$ was added to $1 \mathrm{~mL}$ toluene solution of seeds $(0.05 \mathrm{mg} / \mathrm{mL}$, oblate platelet, hexagonal platelet or hexagonal prism, $m_{\text {unimer }} / m_{\text {seed }}$ is $2: 1$ in the seeded growth experiments for concentric platelet and prism). Then the solution was heated at $80^{\circ} \mathrm{C}$ with stirring for $1 \mathrm{~h}$ before the solution was allowed to cool down naturally to $20^{\circ} \mathrm{C}$. 


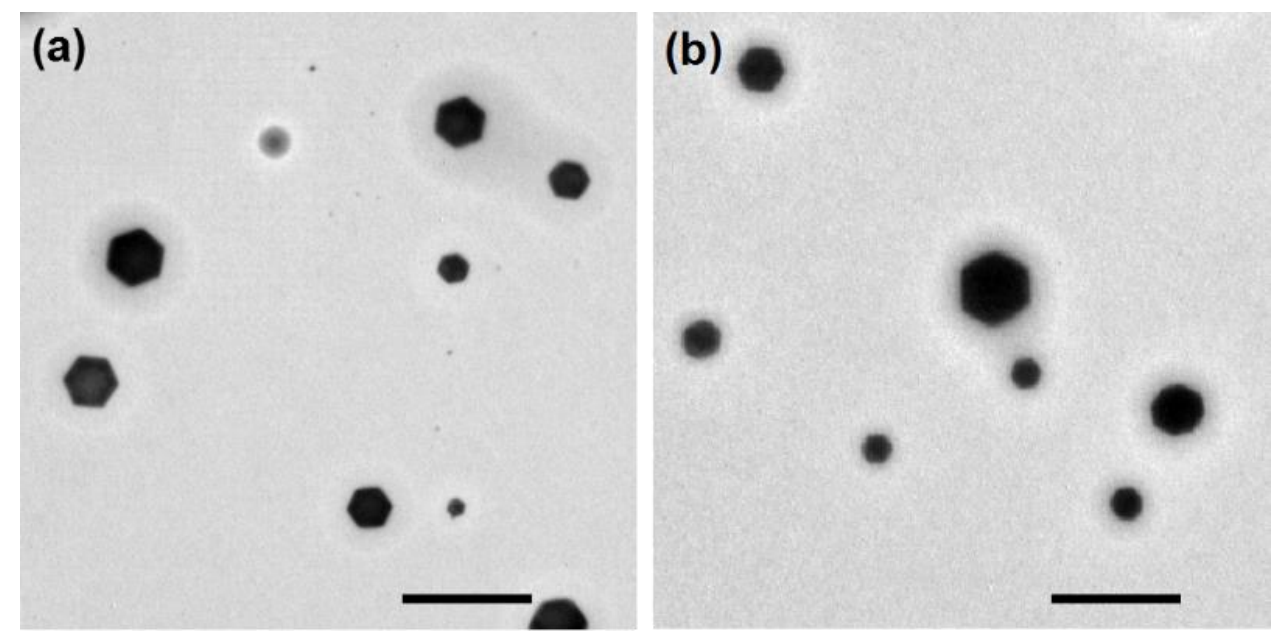

Figure S1. TEM images of P-E-P/TPP hexagonal platelets and uninteracted free polymer chains obtained by adding excessive P-E-P to prepare P-E-P/TPP inclusion complex: (a) EO/TPP =1.5, (b) EO/TPP =1.7. Scale bars are $2 \mu \mathrm{m}$.

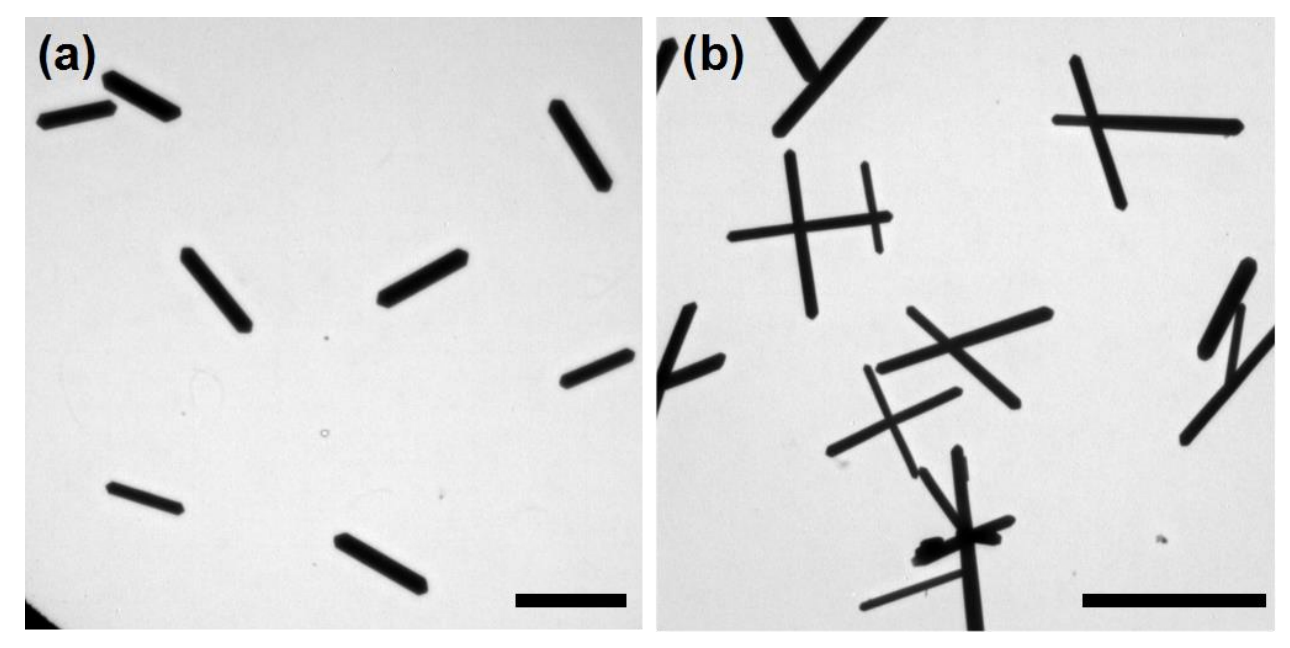

Figure S2. TEM images of hexagonal column-like crystals obtained by adding excessive TPP to prepare P-E-P/TPP inclusion complex: (a) EO/TPP $=0.9$, (b) EO/TPP $=1.1$. Scale bars are $10 \mu \mathrm{m}$.
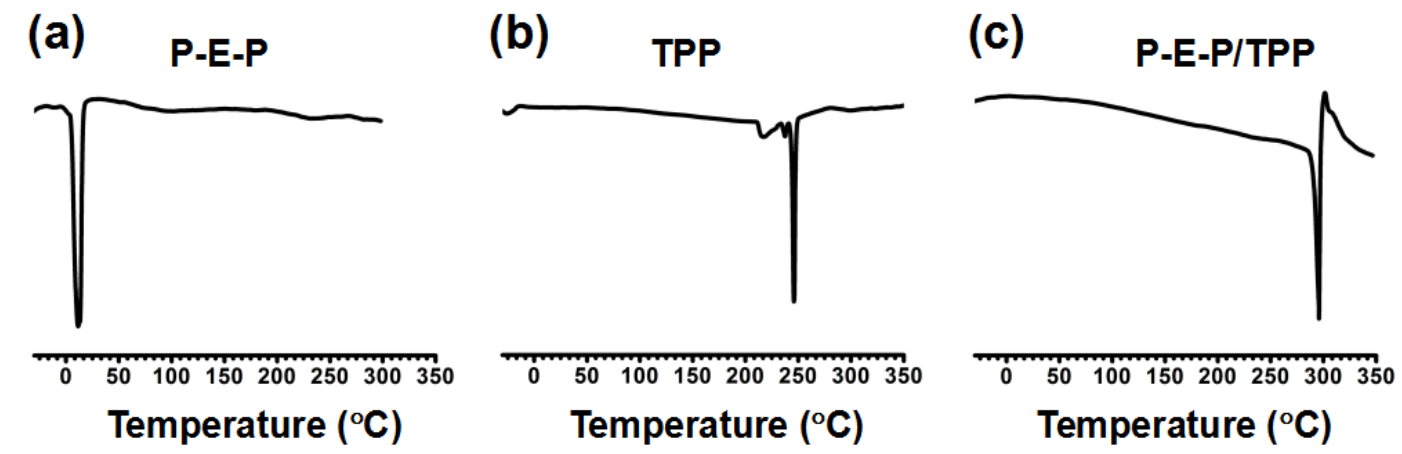

Figure S3. DSC traces of (a) P-E-P, (b) TPP solid and (c) dried P-E-P/TPP platelets (EO/TPP = 1.3:1). 


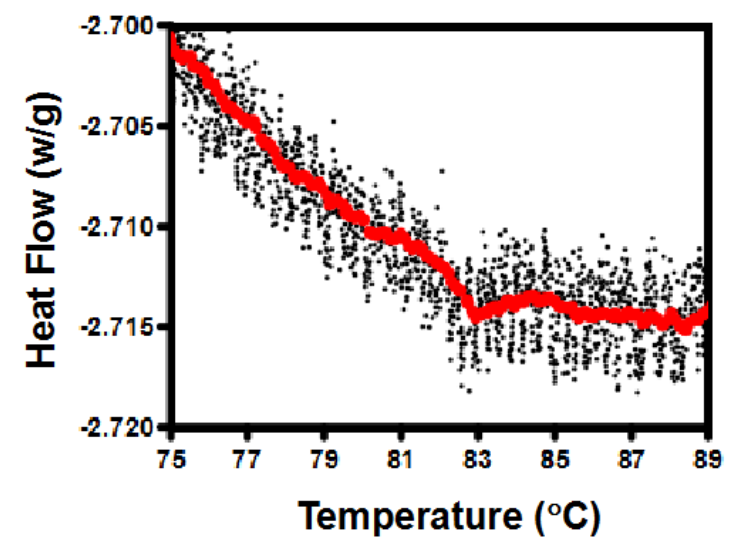

Figure S4. High-resolution DSC of P-E-P/TPP platelets in toluene.
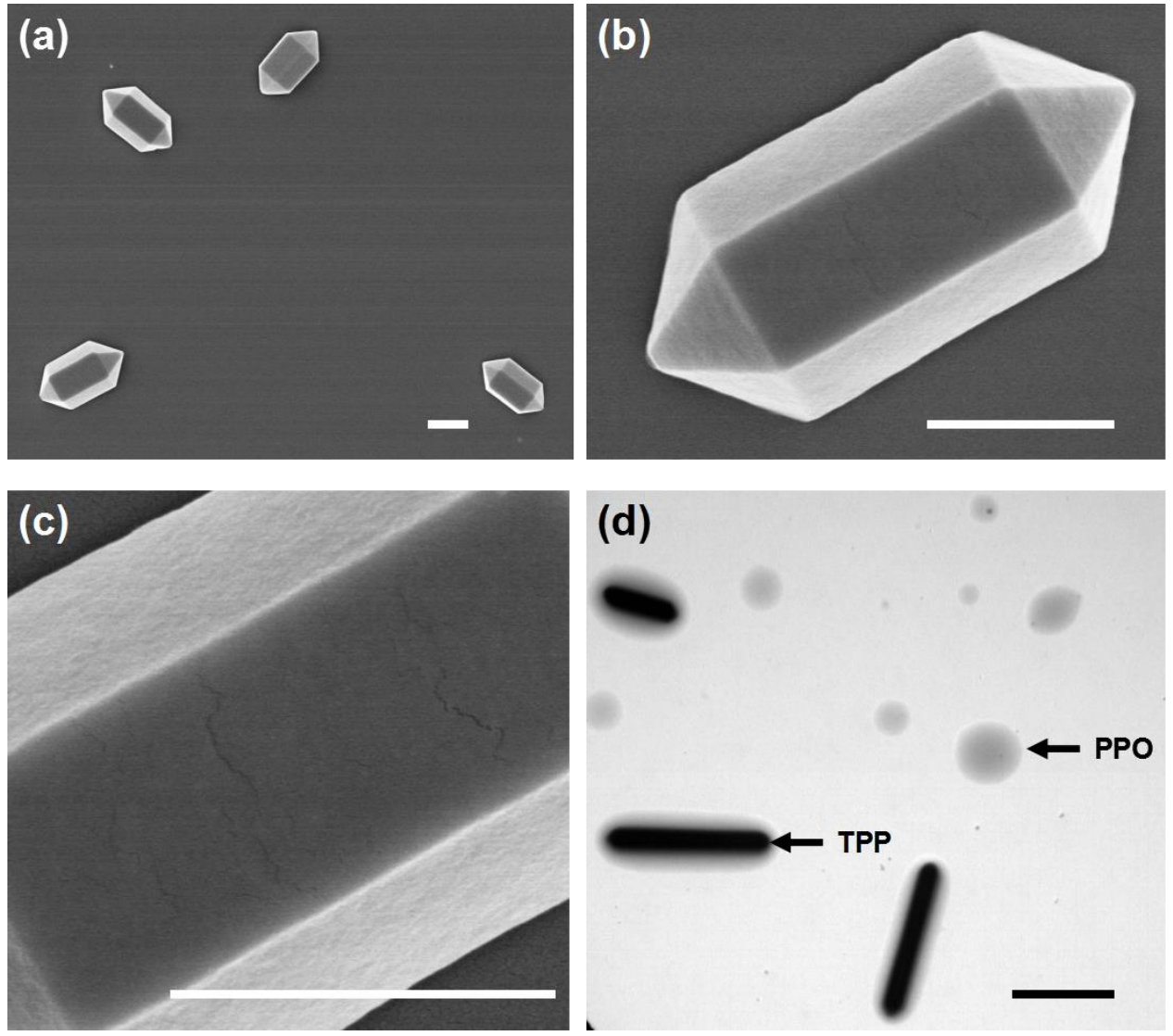

Figure S5. (a) SEM image, and (b, c) zoomed-in SEM images of PEO $25 / \mathrm{TPP}$ hexagonal prisms. (d) TEM image of the product from $\mathrm{PPO}_{25}$ and TPP (indicated by arrows). Scale bars are $1 \mu \mathrm{m}$ in SEM images and $10 \mu \mathrm{m}$ in TEM image. 


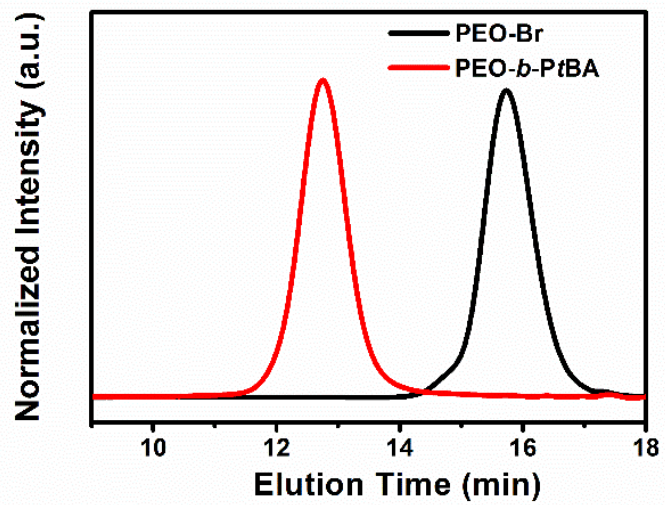

Figure S6. Normalized GPC refractive index (RI) traces of $\mathrm{PEO}_{25}-\mathrm{Br}$ (black) and $\mathrm{PEO}_{25}-b-\mathrm{P} t \mathrm{BA} 92$ diblock copolymer (red). THF was used as the eluent and the concentration was $10 \mathrm{mg} / \mathrm{mL}$.

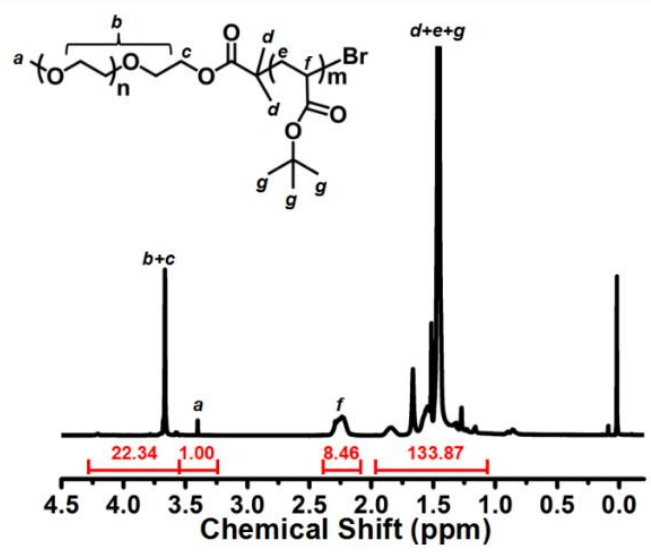

Figure S7. ${ }^{1} \mathrm{H}$ NMR spectrum of the $\mathrm{PEO}_{25}-b$ - $\mathrm{P} t \mathrm{BA} 92$ diblock copolymer ( $\mathrm{CDCl}_{3}$ as solvent).

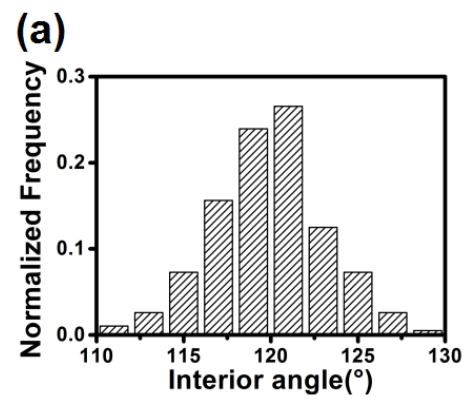

(b)

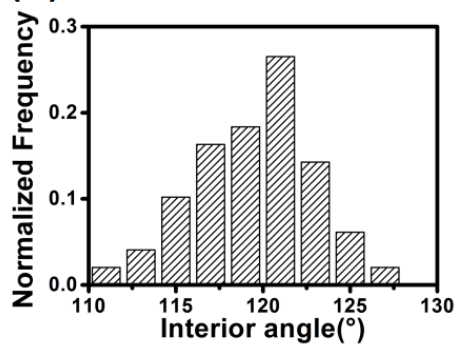

(c)

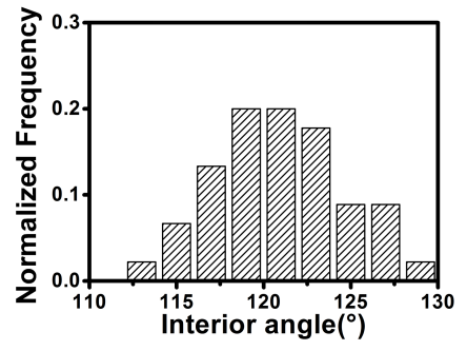

Figure S8. Contour interior angle distributions of (a) P-E-P/TPP hexagonal platelets (b) BA-E/TPP hexagonal platelets and (c) D-B/TPP hexagonal platelets. Detailed data are listed in Table S1. 

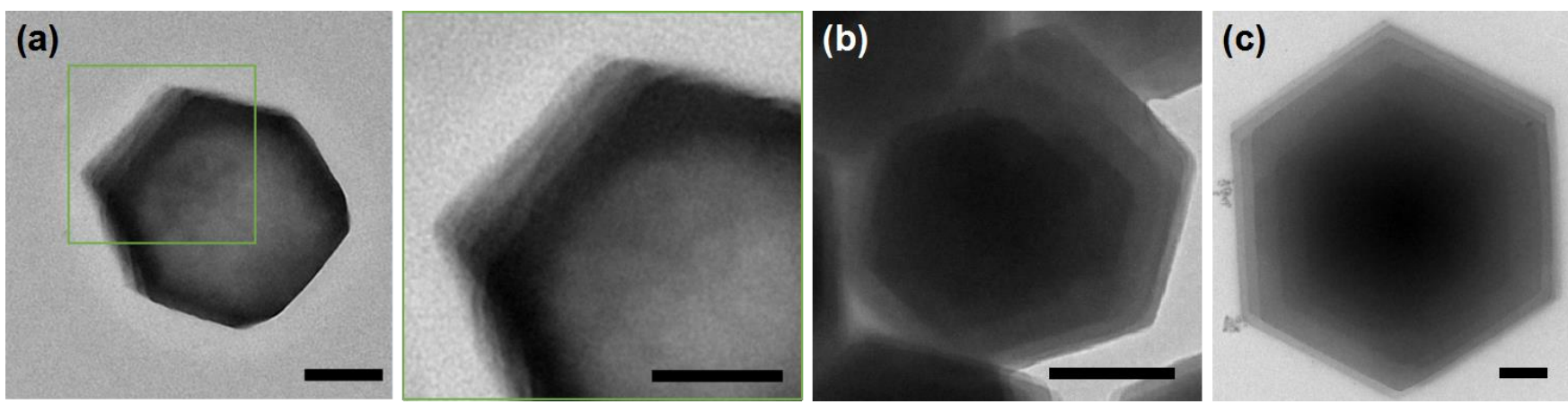

Figure S9. TEM images of (a) slightly tilted P-E-P/TPP platelet (the multilayered structures could be observed from the edge); (b) D-B/TPP platelet showing screw dislocation pattern; (c) BA-E/TPP platelet showing screw dislocation pattern. Scale bars are $200 \mathrm{~nm}$.

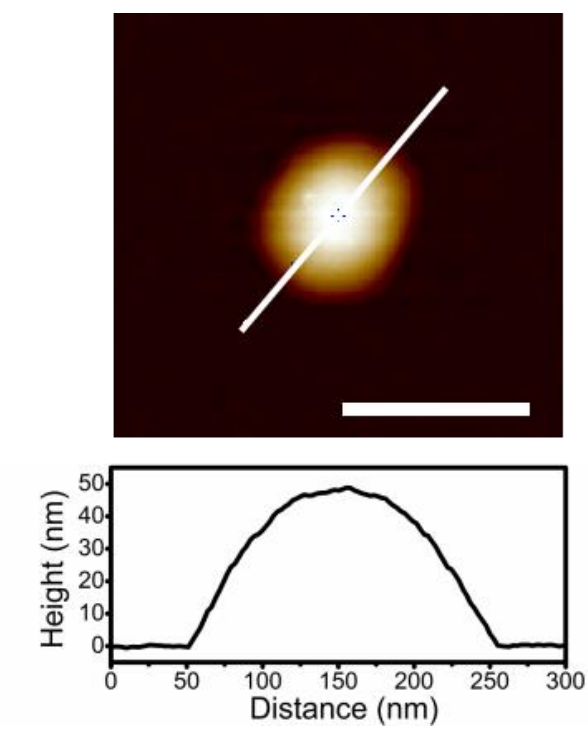

Figure S10. AFM topographic and height profile image of P-E-P/TPP oblate platelet. Scale bar is 200 nm.

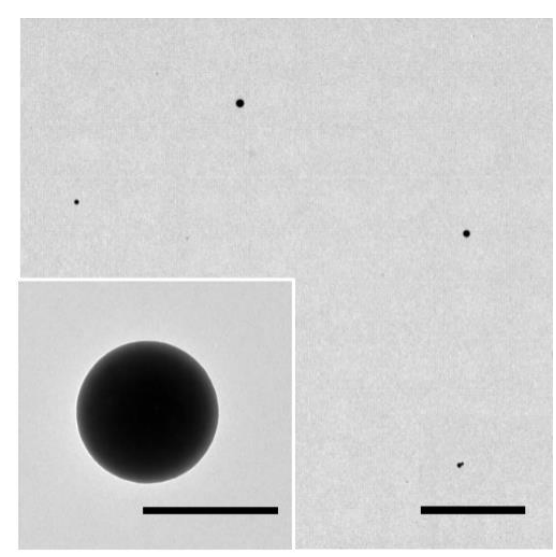

Figure S11. TEM images of P-E-P/TPP oblate platelets from P-E-P/TPP oblate platelets solution heated at $80{ }^{\circ} \mathrm{C}$ for $1 \mathrm{~h}$. Scale bars are $2 \mu \mathrm{m}$ and $200 \mathrm{~nm}$ in the inset. 


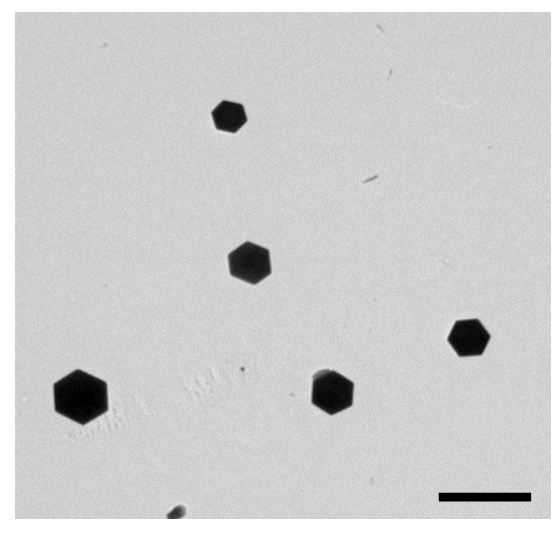

Figure S12. TEM image of non-uniform P-E-P/TPP hexagonal platelets formed from adding excessive amount of P-E-P/TPP unimers in the step of seeded growth of P-E-P/TPP unimers to platelet seeds with $m_{\text {unimer }} / m_{\text {seed }}$ of $4: 1$. Scale bar is $2 \mu \mathrm{m}$.
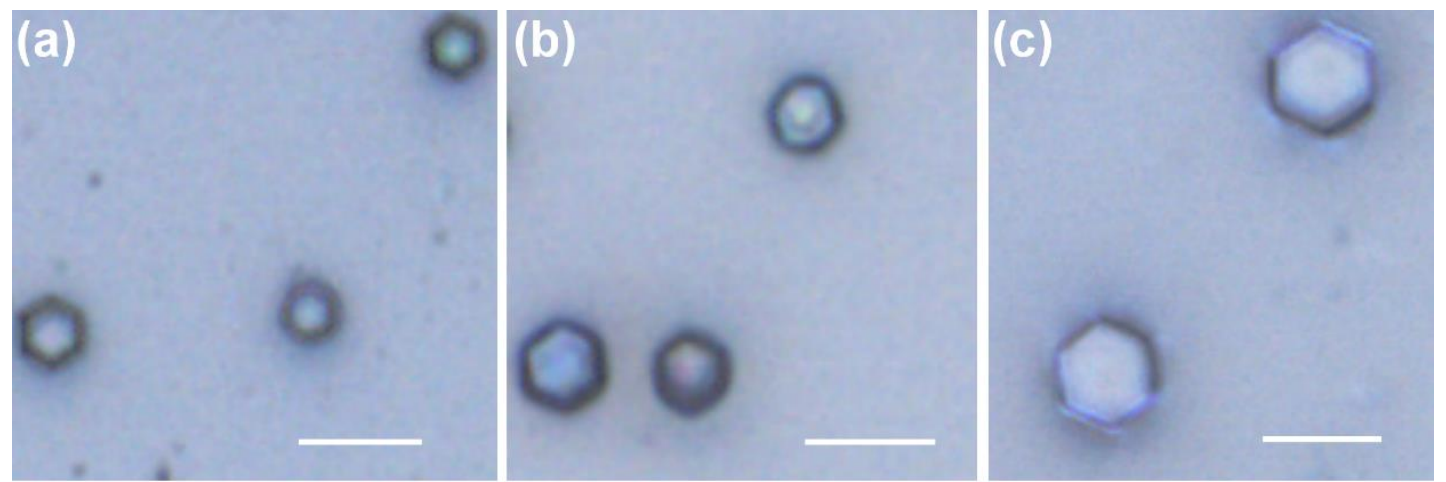

Figure S13. Optical images of P-E-P/TPP hexagonal platelets in toluene solution formed from seeded growth of P-E-P/TPP unimers to P-E-P/TPP seed platelets with $m_{\text {unimer }} / m_{\text {seed }}$ of (a)1:1, (b) 2:1 and (c) 4:1. Scale bars are $3 \mu \mathrm{m}$.

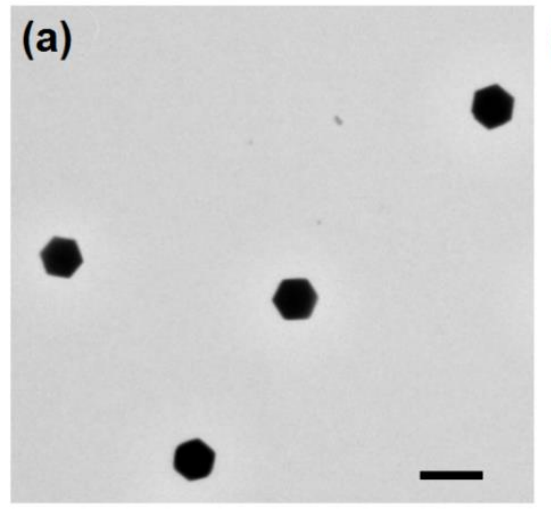

(b)
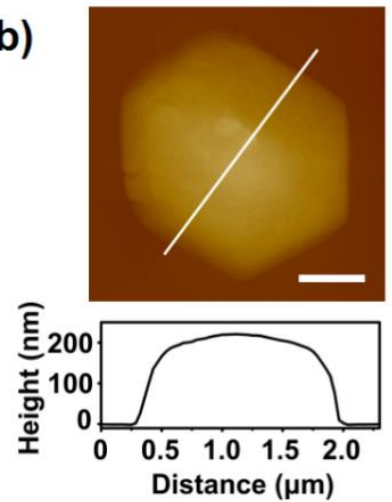

Figure S14. TEM image, corresponding AFM topographic and height profile image of hexagonal platelets prepared from seeded growth of P-E-P/TPP unimers to pre-existing P-E-P/TPP hexagonal 
platelets (side length $677 \mathrm{~nm}$, height $147 \mathrm{~nm}$ ), with a final $m_{\text {unimer }} / m_{\text {seed }}$ ratio of $2: 1$. Scale bars are $2 \mu \mathrm{m}$ in TEM image and $500 \mathrm{~nm}$ in AFM image. 
Table S1. Contour interior angle distributions of P-E-P/TPP hexagonal platelets, BA-E/TPP hexagonal platelets and D-B/TPP hexagonal platelets.

\begin{tabular}{|l|c|c|c|c|c|}
\hline & $A_{n}\left(^{\circ}\right)$ & $A_{w}\left({ }^{\circ}\right)$ & $A_{w} / A_{n}$ & $\sigma\left(^{\circ}\right)$ & $\sigma / A_{\mathrm{n}}$ \\
\hline P-E-P/TPP hexagonal platelet & 119.8 & 120.0 & 1.00 & 3.8 & 0.03 \\
\hline BA-E/TPP hexagonal platelet & 119.8 & 120.0 & 1.00 & 3.6 & 0.03 \\
\hline D-B/TPP hexagonal platelet & 120.1 & 120.2 & 1.00 & 4.4 & 0.04 \\
\hline
\end{tabular}

Table S2. Unit-cell parameters and volume of TPP and the three inclusion complexes (P-E-P/TPP, BAE/TPP and D-B/TPP).

\begin{tabular}{|c|c|c|c|c|}
\hline & Space group & $a, b(\AA)$ & $c(\AA)$ & $V\left(\AA^{3}\right)$ \\
\hline TPP & $P 6_{3} / m$ & $11.4925(3)$ & $10.1518(4)$ & $1161.1(9)$ \\
\hline P-E-P/TPP & $P 6_{3} / m$ & $11.5586(6)$ & $9.9433(7)$ & $1150.5(2)$ \\
\hline BA-E/TPP & $P 6_{3} / m$ & $11.5294(3)$ & $10.0318(5)$ & $1154.8(6)$ \\
\hline D-B/TPP & $P 63 / m$ & $11.5475(8)$ & $10.0026(4)$ & $1155.1(2)$ \\
\hline
\end{tabular}

Table S3. Contour length distributions of P-E-P/TPP oblate platelets and P-E-P/TPP platelets prepared by the seeded growth of P-E-P/TPP unimers to P-E-P/TPP platelet seeds in toluene.

\begin{tabular}{|c|c|c|c|c|c|}
\hline$m_{\text {unimer }} / m_{\text {seed }}$ & $L_{n}(\mathrm{~nm})$ & $L_{w}(\mathrm{~nm})$ & $L_{w} / L_{n}$ & $\sigma(\mathrm{nm})$ & $\sigma / L_{n}$ \\
\hline $0: 1^{\mathrm{a}}$ & 180 & 185 & 1.03 & 31 & 0.17 \\
\hline $0.2: 1^{\mathrm{b}}$ & 248 & 253 & 1.02 & 33 & 0.13 \\
\hline $0.5: 1^{\mathrm{b}}$ & 489 & 492 & 1.01 & 36 & 0.07 \\
\hline $1: 1^{\mathrm{b}}$ & 677 & 682 & 1.01 & 59 & 0.09 \\
\hline $2: 1^{\mathrm{b}}$ & 910 & 917 & 1.01 & 58 & 0.06 \\
\hline $2: 1^{\mathrm{c}}$ & 916 & 922 & 1.01 & 57 & 0.06 \\
\hline $4: 1^{\mathrm{d}}$ & 1230 & 1238 & 1.01 & 58 & 0.05 \\
\hline
\end{tabular}

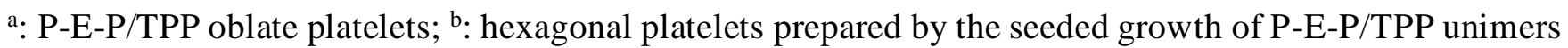
to P-E-P/TPP oblate platelet seeds; ${ }^{c}$ : hexagonal platelets prepared by the seeded growth of P-E-P/TPP unimers to pre-existing P-E-P/TPP hexagonal platelets (side length $677 \mathrm{~nm}$, height $147 \mathrm{~nm}$ ), with a final

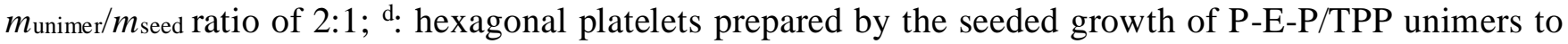
pre-existing P-E-P/TPP hexagonal platelets (side length $910 \mathrm{~nm}$, height $216 \mathrm{~nm}$ ), with a final $m_{\text {unimer }} / m_{\text {seed }}$ ratio of $4: 1$. 
Table S4. Contour height distributions of P-E-P/TPP oblate platelets and P-E-P/TPP platelets prepared by the seeded growth of P-E-P/TPP unimers to P-E-P/TPP platelet seeds in toluene.

\begin{tabular}{|c|c|c|c|c|c|}
\hline$m_{\text {unimer }} / m_{\text {seed }}$ & $H_{n}(\mathrm{~nm})$ & $H_{w}(\mathrm{~nm})$ & $H_{w} / H_{n}$ & $\sigma(\mathrm{nm})$ & $\sigma / H_{n}$ \\
\hline $0: 1^{\mathrm{a}}$ & 45 & 49 & 1.09 & 13 & 0.29 \\
\hline $0.2: 1^{\mathrm{b}}$ & 57 & 58 & 1.02 & 2 & 0.04 \\
\hline $0.5: 1^{\mathrm{b}}$ & 99 & 100 & 1.01 & 2 & 0.02 \\
\hline $1: 1^{\mathrm{b}}$ & 146 & 147 & 1.01 & 2 & 0.01 \\
\hline $2: 1^{\mathrm{b}}$ & 216 & 218 & 1.01 & 3 & 0.01 \\
\hline $2: 1^{\mathrm{c}}$ & 210 & 212 & 1.01 & 4 & 0.02 \\
\hline $4: 1^{\mathrm{d}}$ & 251 & 254 & 1.01 & 7 & 0.03 \\
\hline
\end{tabular}

$\mathrm{a} \sim \mathrm{d}$ : the same as those for Table $\mathrm{S} 2$.

Table S5. Calculated volume distributions of P-E-P/TPP platelets prepared by the seeded growth of PE-P/TPP unimers to P-E-P/TPP platelet seeds in toluene.

\begin{tabular}{|c|c|c|c|}
\hline$m_{\text {unimer }} / m_{\text {seed }}$ & $V_{n}\left(10^{8} \mathrm{~nm}^{3}\right)$ & $V_{w}\left(10^{8} \mathrm{~nm}^{3}\right)$ & $V_{w} / V_{n}$ \\
\hline $0.2: 1^{\mathrm{b}}$ & 0.0911 & 0.0965 & 1.06 \\
\hline $0.5: 1^{\mathrm{b}}$ & 0.6150 & 0.6289 & 1.02 \\
\hline $1: 1^{\mathrm{b}}$ & 1.7384 & 1.7763 & 1.02 \\
\hline $2: 1^{\mathrm{b}}$ & 4.6470 & 4.7625 & 1.02 \\
\hline $2: 1^{\mathrm{c}}$ & 4.5778 & 4.6821 & 1.02 \\
\hline $4: 1^{\mathrm{d}}$ & 9.8656 & 10.1138 & 1.03 \\
\hline
\end{tabular}

b d: the same as those for Table $\mathrm{S} 2$.

Table S6. DLS characteristics of P-E-P/TPP oblate platelets and P-E-P/TPP hexagonal platelets prepared by the seeded growth of P-E-P/TPP unimers to P-E-P/TPP platelet seeds in toluene.

\begin{tabular}{|c|c|c|c|c|c|c|c|}
\hline$m_{\text {unimer }} / m_{\text {seed }}$ & $0: 1^{\mathrm{a}}$ & $0.2: 1^{\mathrm{b}}$ & $0.5: 1^{\mathrm{b}}$ & $1: 1^{\mathrm{b}}$ & $2: 1^{\mathrm{b}}$ & $2: 1^{\mathrm{c}}$ & $4: 1^{\mathrm{d}}$ \\
\hline$D_{\text {h,app }}(\mathrm{nm})$ & 167 & 237 & 312 & 409 & 517 & 521 & 698 \\
\hline PDI $^{*}$ & 0.15 & 0.11 & 0.12 & 0.14 & 0.22 & 0.22 & 0.28 \\
\hline
\end{tabular}

$\mathrm{a} \sim \mathrm{d}$ : the same as those for Table $\mathrm{S} 2$.

*: these PDI values are not as good as those obtained from the TEM images mentioned in the manuscript, mainly due to the large aspect ratio of these platelets. 


\section{References}

(1) (a) Ema, K.; Yao, H. Some Aspects of Recent Improvements of Temperature-Modulated Calorimeter.

Thermochim. Acta 1997, 305, 157-163; (b) Wang, S.; Tozaki, K.; Hayashi, H.; Inaba, H. Nano-Watt Stabilized DSC and ITS Applications. J. Therm. Anal. Calorim. 2005, 79, 605-613; (c) Aya, S.; Sasaki, Y.; Araoka, F.; Ema, K.; Ishikawa, K.; Emelyanenko, A. V.; Takezoe, H. Observation of Two IsotropicNematic Phase Transitions Near a Surface. Phys. Rev. Lett. 2011, 106, 117801.

(2) Tian, N.-N.; Wang, L.-S.; Jiang, R.-Y. Solubilities of Tris(o-phenylenedioxy)cyclotriphosphazene in Selected Solvents. J. Chem. Eng. Data 2011, 56, 3208-3213. 